Purdue University

Purdue e-Pubs

2010

\title{
Thermal Management of a Soft Starter: Transient Thermal Impedance Model and Performance Enhancements using Phase Change Materials
}

F. Volle

Purdue University

S. V. Garimella

Purdue University, sureshg@purdue.edu

M. A. Juds

Eaton Corporation

Follow this and additional works at: http://docs.lib.purdue.edu/coolingpubs

Volle, F.; Garimella, S. V.; and Juds, M. A., "Thermal Management of a Soft Starter: Transient Thermal Impedance Model and Performance Enhancements using Phase Change Materials" (2010). CTRC Research Publications. Paper 251.

http://dx.doi.org/10.1109/TPEL.2010.2040632

This document has been made available through Purdue e-Pubs, a service of the Purdue University Libraries. Please contact epubs@purdue.edu for additional information. 


\title{
Thermal Management of a Soft Starter: Transient Thermal Impedance Model and Performance Enhancements using Phase Change Materials
}

\author{
Fabien Volle, Suresh V. Garimella, and Mark A. Juds
}

\begin{abstract}
Adverse effects of starting-torque transients and high in-rush currents in induction motors are typically mitigated by employing electronically controlled soft starting voltages through silicon controlled rectifiers (SCRs). However, the heat dissipation in the soft starter must be carefully managed in the design of motor drives. The objective of this study is both to address the heat dissipation in the soft starter by implementing analytical solutions to the heat diffusion equations inside the soft starter, and to investigate the use of a phase change material (PCM)-based heat sink for thermal management of the device. The analytical modelling approach is, however, general, and can be applied to the solution of a range of thermal problems in power electronics. The transient analytical thermal model, based on the thermal quadrupole approach, allows a determination of the transient performance of a soft starter by evaluating the thyristor junction temperature. Predictions from the model are first compared to results obtained using a coupled thermal and electrical model based on a resistance/capacitance network approach. Experimental results obtained with the soft starter connected to a low-voltage 200 hp induction machine are then used to validate the model. Additionally, the performance improvement resulting from the use of a hybrid heat sink (plate fin heat sink immersed in a PCM) is evaluated and compared to a conventional air-cooled heat sink without a PCM under identical conditions.
\end{abstract}

F. Volle* and S. V. Garimella are with the School of Mechanical Engineering, Purdue University, West Lafayette, IN 47907 USA (*corresponding author: vfabien@purdue.edu).

M. A. Juds is with Eaton Corporation, Innovation Center, Milwaukee, WI 53216 USA. 
NOMENCLATURE

\begin{tabular}{|c|c|c|}
\hline$A$ & area & $\mathrm{m}^{2}$ \\
\hline$c$ & specific heat & $\mathrm{J} \mathrm{kg}^{-1} \mathrm{~K}^{-1}$ \\
\hline$d$ & thickness of PCM & $\mathrm{m}$ \\
\hline$D$ & thermal diffusivity & $\mathrm{m}^{2} \mathrm{~s}^{-1}$ \\
\hline$g$ & volume heat source & $\mathrm{m}^{3}$ \\
\hline$h$ & convective heat transfer coefficient & $\mathrm{W} \mathrm{K}^{-1} \mathrm{~m}^{-2}$ \\
\hline$k$ & thermal conductivity & $\mathrm{W} \mathrm{m}{ }^{-1} \mathrm{~K}^{-1}$ \\
\hline$l$ & length & $\mathrm{m}$ \\
\hline$L$ & latent heat & $\mathrm{J} \mathrm{kg}^{-1}$ \\
\hline$p$ & Laplace parameter & $\mathrm{s}^{-1}$ \\
\hline$P$ & power & $\mathrm{W}$ \\
\hline$q^{\prime \prime}$ & heat flux & $\mathrm{W} \mathrm{m} \mathrm{m}^{-2}$ \\
\hline$Q$ & heat transfer rate & $\mathrm{J}$ \\
\hline$r$ & radius & $\mathrm{m}$ \\
\hline$S$ & surface area & $\mathrm{m}^{2}$ \\
\hline$S(t)$ & melting front & $\mathrm{m}$ \\
\hline$t$ & time & S \\
\hline$T$ & temperature & $\mathrm{K}$ \\
\hline$V$ & volume & $\mathrm{m}^{3}$ \\
\hline$w$ & layer thickness & $\mathrm{m}$ \\
\hline$Z_{t h}$ & thermal resistance & ${ }^{\circ} \mathrm{CW}^{-1}$ \\
\hline
\end{tabular}

\section{Greek symbols}

$\begin{array}{ll}\gamma & \text { firing angle } \\ \rho & \text { mass density }\end{array}$

$\mathrm{kg} \mathrm{m}^{-3}$ 


\section{Subscripts}

$\begin{array}{ll}c & \text { copper bus } \\ d & \text { silicon die } \\ f & \text { fluid } \\ h & \text { fin heat sink } \\ j & \text { junction } \\ s & \text { solid }\end{array}$

\section{INTRODUCTION}

Starting a de-magnetised induction motor from standstill is a demanding and complex process. At the instant of switching on the power supply, all the energy necessary to magnetise the motor, to provide the acceleration force, and to supply the kinetic energy of the rotor and load, must be present together with the energy to overcome the mechanical and electrical losses in the motor. Doing this at full supply voltage places considerable stresses on the supply, the motor windings, and the iron cores of the stator and rotor.

Several methods for reducing both current transients and torque pulsations on the shaft can be found in the literature. For instance, direct torque control [1] uses an induction motor model to predict the voltage required to achieve a desired output torque. From current and voltage measurements, it is possible to estimate the instantaneous stator flux and output torque. The model is used to predict the voltage required to drive the flux and torque to the desired values within a fixed time period. Due to nonlinear elements in the control loop, study of the harmonic content of induction motor voltage output controlled by direct torque control is very complex. Kaboli et al. [2] present a probabilistic method to study the harmonic content of the voltage output. The method allows the study of the effect of several parameters (such as stator flux and torque and inverter voltage) on the motor voltage spectrum. In spite of some drawbacks that include harmonic current distortion and lack of re-generation capability [3], another reliable way 
to reduce current transients and torque pulsations on the shaft of an induction motor is to use thyristor rectifiers. By introducing two thyristors back-to-back (or anti-parallel) in each of the phase connections to the motor, and by precisely controlling their turn-on points, an electronic soft starter continuously adjusts the passage of energy from the supply so that it is just sufficient for the motor to perform satisfactorily.

One concern associated with the use of this starting method is the heat dissipation in the soft starter. The conventional approach for managing the transient heat spikes has been to use a solid fin heat sink and a large amount of solid copper to absorb the thermal transients. An alternative cooling approach would be to use a solid/liquid PCM storage unit, which has a high latent heat of melting and which allows the stabilization of the junction temperature at an acceptable level, while at the same time yielding weight and volume savings. Since the thermal transients last only for short periods, the PCM would have time to re-solidify by dissipating heat to the surroundings between pulses.

The present paper addresses the issues of heat dissipation in the soft starter by first implementing analytical solutions to the heat diffusion equations inside the soft starter. The transient analytical thermal model developed allows a determination of the transient performance of a soft starter by evaluating the thyristor junction temperature for any switching time profiles, motor and load combinations, and "ON/OFF" cycles. Even though the variation of the thermal properties of the device material with temperature can also influence the solution of the thermal problem, this effect is not included into the model definition. Some equivalent electrical analog networks are proposed in order to implement the model in a convenient form, and the predictions from the model are first compared against a coupled thermal and electrical model using a resistance/capacitance network approach [4]. Experimental results are then used to validate the model. The thermal performance of a hybrid heat sink is also considered and quantified. The PCM thermal resistance is calculated using exact and approximate solutions for PCM transient melting and re-solidification, and the junction temperatures with different PCMs and under different operating conditions (such as heat input and copper thickness) are compared using the analytical 
thermal model.

\section{ANALYTICAL THERMAL MODEL}

The main purpose of this paper is to implement convenient analytical solutions of the conduction equations in a multilayered system. This system is depicted in Fig. 1 and consists of a single pole assembly of a medium voltage soft starter. The main parts of the system are the bypass contactor (a), the thyristors (b1-b2), the heat sink (c), and the top (d1) and bottom (d2) copper buses. The model is based on the thermal quadrupole formalism [5], which is a very efficient method for linear diffusion modeling, especially when involving multilayered systems with internal sources. For homogeneous media, a linear intrinsic transfer matrix relates the input/output temperature and heat flux after a Laplace transformation and some convenient integral space transforms [6], yielding exact analytical relationships in the transformed space. The main advantage of this kind of formulation is to simplify the representation of multilayered systems by just multiplying the corresponding matrices. The explicit analytical nature of this approach provides a better insight into the solution.

\section{A. Heat transfer inside the SCR}

In the literature, many papers presenting electrical and/or thermal models of power semiconductor devices can be found. Wang et al. [7], describe a systematic design optimization approach for inductors and capacitors in diode front-end rectifiers for voltage source inverters. Examples show that the optimization process - based on a genetic algorithm - can lead to a smaller/lower cost inductor and capacitor design. Juds et al. [4] and Profumo et al. [8] describe heat transfer inside thyristors by linear diffusion and distributed resistor and capacitor (RC) networks and present RC equivalent thermal models. Bruckner and Bernet [9] discuss the difficulties and quantify the limitations of this approach with the example of a three-level voltage source converter with insulated gate bipolar transistors. They compare their calculations to the results of direct junction temperature measurements with an infrared camera.

In this section, an analytical method for calculating the instantaneous junction temperature of a SCR is presented. Unless otherwise specified, the term SCR in this work refers to the 
entire device including the package, and not just the semiconductor. The results refer to the T501 N phase-control thyristor depicted in Fig. 2. The thermal model is based on the following assumptions:

- The duration of the current transient allows the heat to diffuse from the silicon die to other confining layers.

- The duration of the current transient does not allow any significant change of the heat sink temperature (which means that we can impose the cathode and/or the anode temperature).

- The variations of the silicon and copper thermal conductivities and specific heat due to temperature are neglected.

Given the above assumptions, the device junction temperature is obtained by the following different steps. First, the heat transfer equation is analytically solved in each part of the SCR (i.e., in each copper and silicon cylinder) using the thermal quadrupole formalism. The detailed analysis is presented in the next section for the silicon die; the solution for the copper layers takes a similar form. Second, knowing that the cylinders have different lateral extensions, constriction resistances are calculated in order to link the different parts together. Last, the theoretical transient thermal resistance is used to estimate the different contact resistances inside the SCR (on each side of the silicon die) and to validate the model.

1) Temperature inside the silicon die: The silicon die, of thickness $w_{d}$, surface area $S_{d}$ and thermal diffusivity $D_{d}$, is subject to internal heating characterized by a volume heat source $g_{d}$ that can vary in time and space. Whatever the boundary conditions, the thermal behavior of the system is described by the following governing equation

$$
\frac{\partial^{2} T}{\partial z^{2}}+\frac{1}{k_{d}} g_{d}(z, t)=\frac{1}{D_{d}} \frac{\partial T}{\partial t}
$$

and initial condition $T=T_{0}$ for $t=0 . k_{d}$ is the thermal conductivity of the silicon die.

Laplace-transforming this equation yields

$$
\frac{d^{2} \theta}{d z^{2}}+\frac{1}{k_{d}} G_{d}(z, p)+\frac{1}{D_{d}} T_{0}=\frac{p}{D_{d}} \theta
$$


where $G_{d}$ is the Laplace transform of $g_{d}$ and $p$ is the Laplace parameter. The solution of this equation can be cast in the following form:

$$
\theta=K_{1} \sinh (\alpha z)+K_{2} \cosh (\alpha z)+y(z, p)
$$

with $\alpha^{2}=p / D_{d}$ and $y(z, p)$ being particular solutions of Eqn. (2) which can be calculated using, for example, the following series expansion:

$$
y(z, p)=\frac{1}{\rho_{d} c_{d} p}\left[G_{d}(z, p)+\frac{D_{d}}{p} \frac{\partial^{2} G_{d}}{\partial z^{2}}+\frac{D_{d}^{2}}{p^{2}} \frac{\partial^{4} G_{d}}{\partial z^{4}}+\ldots\right]+\frac{1}{p} T_{0}
$$

where $\rho_{d}$ is the mass density and $c_{d}$ the specific heat of the medium. $K_{1}$ and $K_{2}$ are integration constants calculated using the output quantities $\theta_{\text {out }}$ and $\Phi_{\text {out }}$, that represent the Laplace transforms of temperature and flux at $z=w_{d}$, respectively. The input quantities $\theta_{i n}$ and $\Phi_{\text {in }}$ related to $z=0$ can be derived from this calculation and the resulting expressions can be cast in the following form (thermal quadrupole formalism or ABCD transmission parameters):

$$
\left[\begin{array}{c}
\theta_{\text {in }} \\
\Phi_{\text {in }}
\end{array}\right]=\left[\begin{array}{cc}
A & B \\
C & D
\end{array}\right]\left[\begin{array}{c}
\theta_{\text {out }} \\
\Phi_{\text {out }}
\end{array}\right]-\left[\begin{array}{c}
X \\
Y
\end{array}\right]
$$

where the coefficients of matrix $M_{\text {slab }}$

$$
\begin{aligned}
& A=D=\cosh \left(\alpha w_{d}\right) \\
& B=\frac{1}{k_{d} S_{d} \alpha} \sinh \left(\alpha w_{d}\right) \\
& C=k_{d} S_{d} \alpha \sinh \left(\alpha w_{d}\right)
\end{aligned}
$$

do not depend on the internal source, and where $X$ and $Y$ are, respectively, a voltage source and a current source such that

$$
\begin{gathered}
X=\int_{0}^{w_{d}}\left(\frac{1}{k_{d}} G_{d}(z, p)+\frac{1}{D_{d}} T_{0}\right) \frac{\sinh (\alpha z)}{\alpha} d z \\
Y=\int_{0}^{w_{d}} S_{d}\left(G_{d}(z, p)+\rho_{d} c_{d} T_{0}\right) \cosh (\alpha z) d z
\end{gathered}
$$

An impedance network, which is strictly equivalent to Eqn. (5), is shown in Fig. 3 with

$$
Z_{1}=\frac{A-1}{C} ; \quad Z_{2}=\frac{D-1}{C} ; \quad Z_{3}=\frac{1}{C}
$$

The same result is obtained for each part of the SCR (with $X=0$ and $Y=0$ in the absence of a heat source) leading to a very convenient way to calculate temperature inside the SCR. Indeed, the SCR being a multilayered medium composed of $N$ layers (see Fig. 4), a representation of 
this multilayered medium is obtained by just multiplying the corresponding quadrupole matrices (the outputs of one part being the inputs to the following one).

However, as previously mentioned, the different parts of the SCR (including the package) have different lateral extensions and constriction resistances must be determined in order to take this into account and to link the parts together.

The derivation of the constriction resistances is detailed in [5]. One can show that the constriction resulting from heat transfer between medium 1 (lateral extension $r_{1}$ ) and medium 2 (lateral extension $r_{2}$ ) can be characterized by the following matrix

$$
M_{\text {constr }}=\left[\begin{array}{cc}
1 & \sum_{1}^{\infty} F_{n} \\
0 & 1
\end{array}\right]
$$

where

$$
F_{n}=\frac{4 J_{1}^{2}\left(\alpha_{n} r_{1}\right)}{k \pi r_{1}^{2} r_{2}^{2} \alpha_{n}^{2} \gamma_{n} J_{0}^{2}\left(\alpha_{n} r_{1}\right)}
$$

with $\gamma_{n}^{2}=\alpha_{n}^{2}+\sigma^{2}\left(\sigma^{2}=p / D_{d}\right.$ and $\alpha_{n}$ being a solution of $\left.J_{1}\left(\alpha_{n} r_{2}=0\right)\right)$, and where $J_{0}$ and $J_{1}$ are Bessel's functions of the first kind of order 0 and order 1, respectively.

2) Validation of the transient thermal impedance: Knowing the different constriction resistances and each slab thermal quadrupole, the temperature inside the SCR can now be easily calculated. Indeed, if $\theta_{0}$ and $\Phi_{0}$ refer to the anode side and $\theta_{w_{s c r}}$ and $\Phi_{w_{s c r}}$ to the cathode side ( $w_{s c r}$ being the total thickness of the SCR), then these quantities are related by the following relation:

$$
\begin{gathered}
{\left[\begin{array}{c}
\theta_{0} \\
\Phi_{0}
\end{array}\right]=M_{\text {slab } 1} \cdot M_{\text {constr } 1} \ldots M_{s} \cdot M_{\text {constr } s} \ldots M_{\text {slab } N} \cdot M_{\text {constr } N}\left[\begin{array}{c}
\theta_{w_{\text {scr }}} \\
\Phi_{w_{\text {scr }}}
\end{array}\right]} \\
-M_{\text {slab } 1} \cdot M_{\text {constr } 1} \cdots\left[\begin{array}{c}
X \\
Y
\end{array}\right]
\end{gathered}
$$

where $M_{\text {slabi }}$ is the matrix associated with the layer $i(i=1$ to $N), M_{s}$ is the matrix associated to the silicon die and $M_{\text {constri }}$ is the matrix that characterizes constriction from medium $i$ to layer 
$i+1$ (Eqn. (9)). Equation (11) allows a calculation of the junction temperature in the Laplace domain when two boundary conditions are given. The inverse in the time domain is performed using Stehfest's numerical algorithm [10].

This junction temperature, $T_{j}(t)$, is used to calculate the transient thermal resistance of the $\mathrm{SCR}, Z_{t h}(t)$, defined by

$$
Z_{t h}(t)=\frac{T_{j}(t)-T_{c}(t)}{P_{j}(t)}
$$

where $T_{c}(t)$ is the case temperature, and $P_{j}(t)$ is the junction-dissipated power at the time instant t. $P_{j}(t)$ is a function of the threshold voltage, $V_{T O}$, and the slope resistance, $r_{T}[11]$

$$
P_{j}=V_{T O} I+r_{T} I^{2}
$$

For a dissipated power of $1 \mathrm{~W}$ and an imposed case temperature of $0{ }^{\circ} \mathrm{C}$, the junction temperature is equal to the transient thermal resistance, $Z_{t h}(t)$.

For the particular SCR considered in this work (see Fig. 2), the manufacturer's data sheet reports the following thyristor transient thermal resistance $R_{t}$ [11], valid only when the thyristor is continuously "ON", which is not the case during the normal operation of the soft starter (the thyristor being switched "ON" and "OFF" repeatedly):

$$
R_{t}(t)=\sum_{i=1}^{5} R_{i}\left(1-e^{t / T C_{i}}\right)
$$

The incremental thermal resistance $R_{i}$, time constant $T C_{i}$ and elapsed time $t$ are valid for constant heat dissipation and are given in Table 1 and Table 2, depending on the type of cooling that is considered (one-side or both-sides-cooling).

The contact resistances on both sides of the silicon die must be estimated. For this estimation, an Ordinary Least Squares method based on the Levenberg-Marquardt algorithm is used to minimize the quadratic difference between the theoretical thermal resistance (given by Eqn. (14)) and the thermal resistance calculated using Eqs. (11) and (12) in the both-sides-cooling case. In order to validate the estimation, the estimated contact resistances are used to calculate the 
thermal resistance (using Eqs. (11) and (12)) in the cathode-side cooling case, and a comparison is made between theoretical (Eqn. (14)) and calculated thermal resistances.

The thyristor data sheet values for the transient thermal resistance from Eqn. (14) are plotted in Fig. 5 (dashed and solid lines). The values for the junction temperature rise per unit of heat dissipation obtained from Eqn. (11) are also plotted in Fig. 5 (symbols). The values of the contact resistances estimated in the both-sides-cooling case are $0.0031 \mathrm{~m}^{2} \mathrm{~K} / \mathrm{W}$ and $0.015 \mathrm{~m}^{2} \mathrm{~K} / \mathrm{W}$. When using these values to calculate the thermal resistance in the cathode-side cooling case, we see in Fig. 5 that the data lie on top of the line corresponding to the theoretical thermal resistance: this verifies the accuracy of the thermal model.

\section{B. Heat transfer inside the top and bottom copper bus bars}

Heat transfer inside the top and bottom copper bus bars (of length $l_{c}$ and width $w_{c}$ ) is now investigated using the same kind of approach, with heat transfer now being two-dimensional. Heat transfer inside the two copper bus bars differs in terms of the convective boundary condition below the bottom bus. However, the quadrupoles defining each bus do not depend on the boundary conditions, and the temperature inside the bus is then the solution of the following energy equation:

$$
\frac{\partial^{2} T}{\partial x^{2}}+\frac{\partial^{2} T}{\partial z^{2}}+\frac{1}{k_{c}} g_{c}(x, z, t)=\frac{1}{D_{c}} \frac{\partial T}{\partial t}
$$

with the following initial condition:

$$
T=T_{0}
$$

where the $g_{c}$ term represents a volumetric heat source due to the heat dissipated inside the copper bus. The heat dissipation in the current path, $Q_{p}$, is a product of the electrical resistance, $R_{e}$ and the square of the current, $I$

$$
Q_{p}=I^{2} R_{e}
$$

After the double Laplace and Fourier cosine transformation:

$$
\theta\left(\beta_{n}, z, p\right)=\int_{x=0}^{x=l_{c}} \int_{t=0}^{t=\infty} T(x, z, t) \exp (-p t) \cos \left(\beta_{n} x\right) d t d x
$$


the heat equation becomes

$$
\frac{d^{2} \theta}{d z^{2}}-\left(\frac{p}{D_{c}}+\beta_{n}^{2}\right) \theta+F\left(\beta_{n}, z, p\right)=0
$$

with

$$
\begin{aligned}
& F\left(\beta_{n}, z, p\right)=\frac{\varphi}{k_{c} p}+\frac{p}{D_{c}} \int_{x=0}^{x=l_{c}} T_{0} \cos \left(\beta_{n} x\right) d x \\
& \quad+\frac{1}{k_{c}} \int_{x=0}^{x=l_{c}} \int_{t=0}^{t=\infty} g_{c}(x, z, t) \exp (-p t) \cos \left(\beta_{n} x\right) d t d x
\end{aligned}
$$

The first term of the source term $F$ in Eqn. (20) corresponds to the Laplace transform of the boundary condition at $z=0$, while the second term corresponds to the $x$-Fourier cosine transform of the initial temperature. The third term corresponds simply to the double transform of the internal heat source.

With $\gamma_{n}^{2}=\frac{p}{D_{c}}+\beta_{n}^{2}$, it can be shown that a linear relationship between the Laplace and Fourier transforms of temperature and flux can be developed through a thermal quadrupole formulation, Eqn. (21), that has exactly the same form as the solution of the one-dimensional transient heat transfer equation with a source term - see Eqn. (5) - and that is modelled by the same diagram as shown in Fig. 3.

$$
\left[\begin{array}{c}
\theta\left(\beta_{n}, 0, p\right) \\
\Phi\left(\beta_{n}, 0, p\right)
\end{array}\right]=\left[\begin{array}{ll}
A\left(\gamma_{n}\right) & B\left(\gamma_{n}\right) \\
C\left(\gamma_{n}\right) & D\left(\gamma_{n}\right)
\end{array}\right]\left[\begin{array}{c}
\theta\left(\beta_{n}, w_{c}, p\right) \\
\Phi\left(\beta_{n}, w_{c}, p\right)
\end{array}\right]-\left[\begin{array}{c}
X\left(\beta_{n}, p\right) \\
Y\left(\beta_{n}, p\right)
\end{array}\right]
$$

with

$$
\begin{aligned}
& A=D=\cosh \left(\gamma_{n} w_{c}\right) \\
& B=\frac{1}{k_{c} S_{c} \gamma_{n}} \sinh \left(\gamma_{n} w_{c}\right) \\
& C=k_{c} S_{c} \gamma_{n} \sinh \left(\gamma_{n} w_{c}\right)
\end{aligned}
$$

and where $X$ and $Y$ are given by

$$
\begin{aligned}
& X\left(\beta_{n}, p\right)=\int_{0}^{w_{c}} F\left(\beta_{n}, z, p\right) \frac{\sinh (\alpha z)}{\alpha} d z \\
& Y\left(\beta_{n}, p\right)=\int_{0}^{w_{c}} k_{c} F\left(\beta_{n}, z, p\right) \cosh (\alpha z) d z
\end{aligned}
$$

Again, this relation allows the calculation of temperature and flux in the Laplace and Fourier domain, and a double inversion is then used to return to the real time-space domain. 


\section{Fin heat sink}

The heat sink, made out of aluminium, is characterized by its fin number and dimensions. Heat from the top copper bus is dissipated through the heat sink base first (Eqn. (21)), then through its fins (by constriction, Eqn. (9)) and finally through the ambient air along the fins, via a convective heat transfer coefficient, $h$, that is assumed to be constant, see Fig. 6 .

Due to the high conductivity of the heat sink and the small dimensions of the fins, it is possible to reduce the heat transfer problem inside the fin to a one-dimensional problem. The heat exchange with the outside environment is then taken into account by a source term in the heat equation.

Temperature inside the fin is then a solution of the following equation (for $0<z<l_{h}$ )

$$
\frac{\partial^{2} T}{\partial z^{2}}-\frac{h P_{h}}{k_{h} A_{h}}\left(T-T_{\infty}(t)\right)=\frac{1}{D_{h}} \frac{\partial T}{\partial t}
$$

with the associated initial condition $T=T_{0}(z)$. In Eqn. (24), $A_{h}$ is the area of cross-section of the fin and $P_{h}$ designates its perimeter. Application of the Laplace transformation yields

$$
\frac{d^{2} \theta}{d z^{2}}+\frac{1}{D_{h}} T_{0}+\frac{h P_{h}}{k_{h} A_{h}} \theta_{\infty}(p)=\left(\frac{p}{D_{h}}+\frac{h P_{h}}{k_{h} A_{h}}\right) \theta
$$

Equation (25) is similar to Eqn. (2) and gives rise to the same quadrupole as that of the medium with source that was already given by Eqn. (5), with $w_{d}=l_{h}, X=0, Y=0$ and

$$
\alpha^{2}=\left(\frac{p}{D_{h}}+\frac{h P_{h}}{k_{h} A_{h}}\right)
$$

instead of $\alpha^{2}=p / D_{d}$.

\section{Validation of the model}

By linking together all the parts of the system (namely the heat sink, the top copper bus, the SCR and the bottom copper bus), it is now possible to calculate the junction temperature rise associated with the use of the soft starter. This rise in temperature is linked to the copper thickness, the fin efficiency and the value of the input current. The thermal model represents an efficient tool to characterize this dependency: for instance, it is now possible to optimize the 
quantity of copper needed to dissipate a certain amount of energy (in the form of sensible heat) by optimizing the efficiency of the heat sink in order to keep the junction temperature below a critical value.

1) Comparison with a model based on a resistance/capacitance network approach: A first step toward validation of the model is to compare its results with results obtained when using a coupled thermal and electrical time-domain model based on the electrical analogy for thermal resistance, thermal capacitance and airflow. This model [4] includes the medium voltage power circuit, the control algorithms, the induction machine model, and a transient thermal model and has been validated experimentally. This comparison is made in Fig. 7, where junction temperatures obtained using both models are plotted. The good agreement between the curves confirms the validity of the thermal model developed in the present work.

2) Experimental validation of the model: In order to demonstrate the application of the transient analytical thermal model to a real physical system, predictions from the model are experimentally validated using results obtained by performing a 60-minute transient test using a $480 \mathrm{~V}, 60 \mathrm{~Hz}$, 4-pole, $200 \mathrm{hp}$, squirrel-cage, 3-phase induction motor. The starter was run in the full $\mathrm{ON}$ condition where the thyristors were switched with a very small delay angle, $\gamma$, of about four degrees. This resulted in a nearly sinusoidal current of 66.8 amps. J-type thermocouples were placed as close to the thyristors as possible in the top and bottom bus bars. The RMS current was calculated and used as the input to the heat dissipation both inside the SCR and in the copper buses (Eqs. (13) and (17)). A plot of the measured (symbols) and calculated (lines) temperatures is shown in Fig. 8. The very close agreement between the measured and calculated temperatures validates the transient thermal model; the small differences stem from uncertainties in dimensions and thermophysical properties.

It is noted that even though the model has been applied in this work for predicting the instantaneous junction temperature of thyristors during current transients associated with the use of a soft starter, the underlying method can be applied by power electronics engineers to a wide 
range of problems, especially those involving modelling of stacked electronic structures (thermal analysis of high power IGBT modules, for instance).

\section{EXACT AND APPROXIMATE SOLUTIONS FOR CONDUCTION AND PHASE CHANGE}

\section{A. Specifications of the problem}

We now consider a hybrid heat sink (metallic plate fin heat sink immersed in a PCM) of initial temperature $T_{0}$ whose lower surface is suddenly exposed to a heat input $Q$ at time $t=0$ (Fig. 9) during a period of time $t_{\text {heat }}=2 \mathrm{~min}$. Heat is transferred from the base of the fins (surface at $z=b_{h}$ ) and through the fins to the PCM of volume $V_{P C M}$ equal to the volume of PCM needed to store all the heat dissipated during $t_{\text {heat }}$. Before melting starts, a portion of the input heat goes towards raising the temperature of the PCM, while the rest is lost to the environment of temperature $T_{\infty}$ via the top surface of the heat sink (at $z=b_{h}+l_{h}+d=H$ ) where a constant convective heat transfer coefficient $h$ is assumed. Let $T_{m}$ be the melting temperature of the PCM and $L$ the corresponding latent heat. After the PCM temperature reaches $T_{m}$, a portion of the heat is also consumed by phase change.

When melting occurs, the heat storage inside the PCM can be divided into two regions as depicted in Fig. 10. In Region 1, the only heat source is the uniform heat flux at $z=b_{h}$. Here the fin does not influence the melting process and heat is transferred mainly in the $z$-direction. In Region 2, both the surface at $z=b_{h}$ and the fins transfer heat from the source to the phase change material. The melting process near the fins can be divided into three stages: first, pure conduction from the surface at $z=b_{h}$ and the fins, then conduction from the fins with some natural convection from the surface, and finally, only natural convection from the fins. However, the fins tend to decrease natural convection from the surface due to the decreasing temperature gradient in the liquid.

Several assumptions are made in order to simplify the non-linear heat transfer problem:

1. The temperature distribution of the thin fins is considered to be one-dimensional in the $z$-direction. 
2. The fins have $100 \%$ efficiency. This implies that in Region 1 and Region 2 , the heat is transferred from the surface to the solid-liquid interface one-dimensionally, in the $z$-direction and in the $x$-direction, respectively.

3. The sensible heat of liquid PCM is assumed to be negligible. The latent heat of fusion is assumed to be the principal mode of energy storage.

4. The main heat transfer mode is assumed to be conduction in liquid PCM. Natural convection in liquid PCM is assumed to be negligible.

5. The thermophysical properties for the phase change material and for the fins are assumed to be constant.

6. Liquid and solid phases have identical density $\rho$.

Let $k_{s}, \kappa_{s}, c_{s}$ denote the thermal conductivity, diffusivity and specific heat (at constant pressure) of the solid material, respectively, and $k_{l}, \kappa_{l}, c_{l}$ the corresponding properties of the liquid material after melting. Let $S(t)$ represent the melting front separating the liquid and solid phases. Under these conditions, a one-dimensional analysis of the problem is appropriate and the temperature of the base (i.e., temperature at the metal/PCM interface) may be expressed as $T_{s} \equiv T_{s}(z, t)$ for the solid and $T_{l} \equiv T_{l}(z, t)$ for the liquid and must satisfy the following governing equations:

$$
\begin{array}{ll}
\frac{\partial^{2} T_{l}}{\partial z^{2}}=\frac{1}{\kappa_{l}} \frac{\partial T_{l}}{\partial t}, & 0 \leq z \leq S(t) \\
\frac{\partial^{2} T_{s}}{\partial z^{2}}=\frac{1}{\kappa_{s}} \frac{\partial T_{s}}{\partial t}, & S(t) \leq z \leq d
\end{array}
$$

associated with initial

$$
T_{l}(z, 0)=T_{s}(z, 0)=T_{0}, \quad t=0
$$


and boundary conditions

$$
\begin{gathered}
-k_{l} \frac{\partial T_{l}}{\partial z}=q^{\prime \prime}, \quad z=0 \\
k_{s} \frac{\partial T_{s}}{\partial z}-k_{l} \frac{\partial T_{l}}{\partial z}=\rho L \frac{d S(t)}{d t}, \quad z=S(t) \\
T_{l}(z, t)=T_{s}(z, t)=T_{m}, \quad z=S(t) \\
-k_{s} \frac{\partial T_{s}}{\partial z}=h\left[T_{s}(d, t)-T_{0}\right], \quad z=d \\
S(t)=0, \quad t<t_{m}
\end{gathered}
$$

where $t_{m}$ is the time needed for the PCM to heat up to the melting temperature $T_{m}$. The problem of melting as specified by Eqs. (29)-(31) is nonlinear due to the fact that the velocity of the melting front is coupled to the temperature via Eqn. (31b).

In Table 3, the thermophysical properties of selected materials used for the simulations are specified. Since some properties of $\mathrm{Bi} / \mathrm{In} / \mathrm{Sn}$ were not available, a weighted average of the properties for the constituent elements was used as also done in [12]. Moreover, owing to the poor thermal conductivity of triacontane, its transient thermal performance has been improved by introducing an aluminum foam insert of porosity $\epsilon=0.8$ into the PCM. Thermal equilibrium was assumed at the interface between the solid foam and the fluid matrix. Also the contact at the interface between the foam and the heat source was assumed to be perfect (no contact resistance). The effective thermal capacitance and conductivity of the PCM-foam combination are obtained using the model developed in [13]. The metal foam medium has an open-celled structure composed of dodecahedron-like cells which have pentagonal or hexagonal faces. Since the structure is periodic, only a hexagonal unit cell is considered. The edges of the cell are composed of fibers of thickness $2 t$ and there is a lumping of material at points where the fibers intersect. In the model, this lumping is taken into account in the structure via the square of width 2b. According to [13], the effective thermal capacitance and conductivity are given by

$$
\left(\rho C_{p}\right)_{e f f}=\epsilon\left(\rho C_{p}\right)_{f}+(1-\epsilon)\left(\rho C_{p}\right)_{F F}
$$


and

$$
\begin{aligned}
\frac{1}{k_{e f f}} & =\left(\frac{2}{\sqrt{3}}\right)\left(\frac{A_{R} b}{k_{f}+(1+b) \frac{\left(k_{s}-k_{f}\right)}{3}}+\frac{\left(1-A_{R}\right) b}{k_{f}+\left(\frac{2}{3}\right) b\left(k_{s}-k_{f}\right)}\right. \\
& \left.+\frac{\frac{\sqrt{3}}{2}-b}{k_{f}+\left(\frac{4 A_{R}}{3 \sqrt{3}}\right) b\left(k_{s}-k_{f}\right)}\right)
\end{aligned}
$$

where $\epsilon$ is the foam porosity and $b$ is given by

$$
b=\frac{-A_{R}+\sqrt{A_{R}^{2}+\frac{2}{\sqrt{3}}(1-\epsilon)\left(2-A_{R}\left(1+\frac{4}{\sqrt{3}}\right)\right)}}{\frac{2}{3}\left(2-A_{R}\left(1+\frac{4}{\sqrt{3}}\right)\right)}
$$

The "area ratio" $A_{R}$ is defined as $A_{R}=t / b$ and a value of $A_{R}=0.09$ was used (as suggested in [13]).

\section{B. Sensible heating}

In this section, exact and approximate solutions for the temperature distribution inside the PCM before melting are summarized. These temperature profiles constitute the basis for the subsequent analysis of melting in the next section, and can be used to estimate the thermal resistance of the metal heat sink (without PCM).

1) Volume of PCM: From the assumptions presented above, the total volume of PCM needed to store all the heat dissipated during a duration $t_{\text {heat }}$ is calculated through an energy balance according to

$$
V_{P C M}=\frac{Q t_{\text {heat }}}{\rho\left(L+C_{p}\left(T_{m}-T_{0}\right)\right)}
$$

This volume can be used either to calculate the PCM thickness $d$ by dividing $V_{P C M}$ by the surface area, $A$ (equal to the external top surface of the heat sink), or to calculate the surface area corresponding to a new heat sink by dividing $V_{P C M}$ by $d$ when $d$ is fixed. In both cases, $d$ allows the calculation of the temperature at the heat sink/PCM interface. 
2) Exact and approximate temperatures: In the absence of melting $(S(t) \equiv 0)$, Eqs. (29)(31a), together with the convective heat transfer boundary condition (31d), can be solved by Laplace transforms, yielding

$$
\frac{T_{s}(z, t)-T_{0}}{Q}=\frac{d-z}{k_{s}}+\frac{d}{k_{s}}\left\{\frac{1}{B i}-2 \sum_{n=1}^{\infty} \frac{\exp \left(\frac{-\lambda_{n}^{2} k t}{d^{2}}\right) \cos \left(\frac{\lambda_{n} z}{d}\right)}{\lambda_{n}\left(\lambda_{n}+\sin \lambda_{n} \cos \lambda_{n}\right)}\right\}
$$

Here, the nondimensional heat transfer coefficient, $B i$, is the Biot number defined as $B i=h d / k_{s}$ and $\lambda_{n}$ are the positive roots of the transcendental equation

$$
\lambda_{n} \tan \lambda_{n}=B i
$$

Although the solution (36) to the temperature distribution is exact, it involves the evaluation of the sum of an infinite series, which is non-trivial. An approximate but much simpler solution to the problem can be obtained through a lumped body analysis valid for $B i \ll 0.1$. The solution for the base temperature is

$$
T(t)-T_{\infty}=(q " / h)\left(1-\exp \left(\frac{-h t}{\rho C_{p} d}\right)\right)
$$

\section{Melting and re-solidification}

1) Melting: The process of melting with a moving boundary is a complex problem. Although approximate analytical solutions are available for a few simplified cases with phase change occurring at constant temperature, numerical methods are commonly used to deal with more complicated cases. If, however, we assume that the sensible heat is negligible compared to latent heat (Ste $\left.=c_{s}\left(T_{m}-T_{0}\right) / L \approx 0\right)$, the concept of "quasi-steady state" may be used to arrive at approximate analytical solutions both for the position of the melting line and the surface temperature of the layer as functions of time. The quasi-steady state approach assumes that the variation of temperature with time is slow during melting such that the temperature gradients both in the molten layer and solid layer are approximately constant, although these may change with time. In our case, only the liquid phase temperature will vary with time, the solid phase being at constant temperature, $T_{m}$, during melting process. Under this assumption, the temperature 
profile in the liquid region at a given time $t$ may be taken as [14]

$$
T_{l}(z, t)=T_{m}+\frac{q^{\prime \prime}}{k_{l}}\{S(t)-z\}, \quad z \leq S(t)
$$

which satisfies both Eqs. (31a) and (31c). Substitution of the above temperature profile into the enthalpy balance Eqn. (31b) (with $\partial T_{s} / \partial z=0$ ) leads to the following equation for $S(t)$

$$
q^{\prime \prime}=\rho L \frac{d S(t)}{d t}
$$

which yields

$$
S(t)=\frac{q "\left(t-t_{m}\right)}{\rho L}
$$

where $t_{m}$ is the time needed for the PCM to heat up to the melt temperature $T_{m}$, obtained by linearising Eqn. (38)

$$
T(t)-T_{\infty} \approx(q " / h)\left(\frac{h t}{\rho C_{p} d}\right)
$$

so that

$$
t_{m}=\frac{\left(T_{m}-T_{0}\right)}{q^{\prime \prime}}\left(\rho C_{p} d\right)
$$

Hence, during quasi-steady state melting, the total time $t_{c}$ needed for the layer of PCM of thickness $d$ to completely melt upon heating from the initial temperature $T_{0}$ is

$$
t_{c}=t_{m}+\frac{\rho L d}{q^{\prime \prime}}
$$

where $t_{m}$ is given by Eqn. (43). From Eqs. (39) and (41), the base temperature is obtained as

$$
T(t)=T_{m}+\frac{q^{\prime \prime 2}}{\rho k_{l} L}\left(t-t_{m}\right)
$$

By recasting Eqn. (45) suitably, a thermal resistance for the PCM layer, $R_{P C M}(t)$, can be defined

$$
R_{P C M}(t)=\frac{T(t)-T_{m}}{Q}=\frac{d}{k_{l} A} \frac{Q\left(t-t_{m}\right)}{\rho L V_{P C M}}
$$

From expression (46), it can be seen that thermal resistance of the PCM is a product of conduction resistance and the ratio of heat energy input to the maximum available latent heat energy. In the next section, the thermal resistances of the various materials considered in this work, as well as the thermal resistance of the aluminum heat sink, are integrated into the analytical quadrupolebased thermal model in order to calculate the junction temperature. 
It may be noted that solutions obtained from Eqn. (45) are approximate in the sense that the proposed temperature profile (39) does not satisfy the energy equation (29a); it does, however, preserve the energy balance at the melting front, i.e., Eqn. (31b). The obtained solutions, in general, tend to overestimate both the melt depth and the base temperature.

2) Validity of quasi-steady state solutions: As stated previously, the quasi-stationary approach is valid for Ste $\approx 0$, where Stefan number Ste is a dimensionless measure of the degree of superheating in the liquid. Ste depends on $c_{s}, T_{m}-T_{0}$ and $L$ only. However, the applied heat input $Q$ as well as the thickness of the material $d$ influence the range of validity of this approach. Indeed, in the absence of melting, thermal conduction inside the PCM is characterized by a time $t_{0}$ corresponding to the penetration time of the applied heat input (or flux) from the base surface to the top surface $(z=d)$, such that

$$
t_{0} \sim \frac{d^{2}}{\kappa_{s}}
$$

Thus, if $t<t_{0}$, the top surface of the layer is yet to be affected by the penetration of heat flow such that $T_{s}\left(z=d, t=t_{0}\right)=T_{0}$. As a result, the temperature distribution across the PCM is identical to that for a semi-infinite medium with all of the heat consumed in raising the temperature of the material, which means that the melting process must be occurring at a time $t_{m}>t_{0}$ for the quasi-stationary approach to be valid (sensible heat is negligible compared to the latent heat).

Equation (36) can be used to calculate the penetration time $t_{0}$. Using a series solution [15], it can be shown that $t_{0}=\pi d^{2} / 16 \kappa_{s}$. Since the quasi-steady state approach requires $t_{m}>t_{0}$, it follows from Eq. (43) that the applied heat flux must satisfy

$$
\frac{\left(T_{m}-T_{0}\right)}{q^{\prime \prime}}\left(\rho C_{p} d\right)>\frac{\pi d^{2}}{16 \kappa_{s}}
$$

The above relation leads to the following inequality

$$
q^{\prime \prime}<\frac{16 k_{s}\left(T_{m}-T_{0}\right)}{\pi d}
$$

that may be used to gauge whether or not the quasi-steady state solutions are suitable for a given application. 
3) Resolidification: Since the soft-starter is subjected to repeated pulses (about four $15 \mathrm{~s}$ pulses per hour), resolidification of the melted PCM after the end of an input energy pulse was also investigated, in an effort to determine the allowable frequency of repeated pulses (such that the PCM has enough time to resolidify between pulses). At the end of time $t_{\text {heat }}$, the melt is allowed to cool by convection from the top surface (through convective heat transfer coefficient $h$ ) and the resolidification process occurs. The surface corresponding to the heat source is considered to be perfectly insulated $(Q=0)$ during the resolidification period, providing a conservative estimate of the resolidification time $t_{s}$.

Time $t_{s}$ is estimated using the quasi-stationary approach, which yields

$$
t_{s}=\frac{d^{2}}{2 \kappa_{s} S t e}\left\{1+\frac{2}{B i}\right\}
$$

Equation (50) is valid only if

$$
B i<\frac{2}{\left(\text { Ste } / 2 \lambda^{2}-1\right)}
$$

where $\lambda$ is solution of

$$
\lambda e^{\lambda^{2}} \operatorname{erf}(\lambda)=\frac{S t e}{\sqrt{\pi}}
$$

Moreover, it can be shown from Eqn. (50), that for small Biot number (2 / $B i \gg 1)$

$$
t \approx \frac{\rho L d}{h \Delta T}=\frac{\rho L}{h \Delta T}\left(\frac{V}{A}\right)
$$

Therefore, the time for solidification is the ratio of the energy stored to the heat removal rate. It can be noticed from the above expression that thermal conductivity does not play a role in determining the solidification time under these conditions.

\section{Performance of different PCM-based heat sinks}

In this section, the junction temperature of the SCR referred to as b1 in Fig. 1 is calculated when an aluminum heat sink is used, and then when a hybrid heat sink is considered. In this last case, three different PCMs - whose thermophysical properties are given in Table 3 - are considered for comparison purposes: $\mathrm{Bi} / \mathrm{In} / \mathrm{Sn}$, a triacontane-filled foam and a cerrobend eutectic. The heat generation $Q$ inside the SCR is equal to $3 \mathrm{~kW}$ during $15 \mathrm{~s}$, after which time the heat 
sink is allowed to cool by convection from the top surface (through a convective heat transfer coefficient $\left.h=20 \mathrm{~W} / \mathrm{m}^{2} \mathrm{~K}\right)$. Two values $-w_{1}=0.75 "(\approx 1.9 \mathrm{~cm})$ and $w_{2}=0.35 "(\approx 0.9 \mathrm{~cm})-$ are considered for the thickness of the top copper bus (referred to as d1 in Fig. 1) to investigate whether the use of a hybrid heat sink allows a decrease in the amount of copper that has to be used to keep the junction temperature at an acceptable level. For each material, the condition given by Eqn. (49) is verified so that the quasi-steady state approach is valid. Results are summarized in Table 4. The volume of PCM is calculated using Eqn. (35), and the resolidification time $t_{s}$ is calculated with Eqn. (50). Lastly, the junction temperature is calculated by coupling the thermal quadrupole model with the melting analysis.

Even though the cerrobend eutectic PCM is beneficial in terms of total volume $\left(0.0001 \mathrm{~m}^{3}\right.$ compared with $0.0007 \mathrm{~m}^{3}$ for aluminum), the junction temperature reached is either comparable to the one reached with an aluminum heat sink, or too high when the copper bus thickness is halved to $0.9 \mathrm{~cm}$ : this particular PCM would not be a good choice for the desired application. However, both the metallic alloy $\mathrm{Bi} / \mathrm{In} / \mathrm{Sn}$ and the foam with triacontane yield significantly lower junction temperatures than a solid copper heat sink for which junction temperatures of 103 and $184^{\circ} \mathrm{C}$ are obtained (for copper thicknesses of $w_{1}$ and $w_{2}$, respectively).

A PCM such as $\mathrm{Bi} / \mathrm{In} / \mathrm{Sn}$ is well suited for the application in terms of the junction temperature reached $\left(T_{j}=83.6^{\circ} \mathrm{C}\right.$ for $Q=3 \mathrm{~kW}$ during 15 seconds $)$ and volume $\left(0.0002 \mathrm{~m}^{3}\right.$ instead of $0.0007 \mathrm{~m}^{3}$ for aluminum) even though the required resolidification time ( $\left.t_{s}=675 \mathrm{~min}\right)$ is high. However, a design allowing the PCM to be cooled from both sides would assist in resolidification and would significantly decrease $t_{s}$. The use of a foam PCM is advantageous in terms of resolidification time $\left(t_{s}=9.3 \mathrm{~min}\right)$, even though it leads to higher values of junction temperature $\left(T_{j}=167.6^{\circ} \mathrm{C}\right)$ when decreasing the top copper bus thickness by a factor 2 : depending on the design and on the maximum admissible temperature of the SCR, the foam with triacontane may not be beneficial, but it is still interesting due to its capacity to resolidify quickly between the heat pulses. Simulations have also been performed at double the heat input $(Q=6 \mathrm{~kW}$ during $15 \mathrm{~s})$. It appears that the junction temperatures $\left(T_{j}\right)$ in each case increase, but for the $\mathrm{Bi} / \mathrm{In} / \mathrm{Sn}$ 
system, the new junction temperature is still acceptable at $101.3^{\circ} \mathrm{C}$, which makes $\mathrm{Bi} / \mathrm{In} / \mathrm{Sn}$ the best candidate for the application among the tested PCMs, and compared with an aluminum heat sink.

These results show that a PCM-based heat sink, if designed so that the resolidification time is acceptable (by cooling the PCM from both sides, for instance) allows an increase in the performance of the soft starter cooling approach. Indeed, when compared with an aluminum heat sink, a hybrid heat sink allows not only a decrease in the total volume of the system (which is beneficial in terms of weight savings), but also a decrease in the junction temperature so that the soft starter efficiency can be improved (by increasing the current that flows through it, for instance).

\section{CONCLUSION}

In this paper, it has been demonstrated that an accurate transient thermal model based on the use of the thermal quadrupole method can be developed in order to study heat transfer in a power electronics system. This model can be used for predicting the instantaneous junction temperature of thyristors during current transients associated with the use of a soft starter. The model has been validated by performing a comparison with results obtained by using distributed resistor and capacitor networks, as well as by comparing against experimental results. The results of the model and its validation show that satisfactory results can be obtained. The main advantages of the model developed with respect to other electrothermal simulation approaches are:

- low computational resource requirements;

- possibility of model optimization for a given type of analysis;

- the explicit analytical form of the solution offers better insights into this solution (easy model parameter extraction, for instance);

- and the thermal model can be used to predict and verify the thermal performance for proposed system performance ratings.

Another advantage of the method is its simplicity of use and its applicability to other problems 
of interest in power electronics, especially problems involving modelling of stacked electronic structures.

In addition, a transient thermal analysis was performed to compare the performance of the currently used aluminum heat sink to that enabled with the use of a phase change material-based heat sink for different materials and two different copper bus thicknesses. Easy-to-use expressions have been developed and three different PCMs were studied to explore possible trade-offs in their use. The computations show the use of PCMs in the thermal management of soft starters to be feasible, but further in-depth analysis is needed to find a PCM or PCM-foam combination which is suitable for $T_{j}$ and $t_{s}$. One exciting possibility is to implement an inverse method, where the actual properties of the desired PCM could be computed based on the criteria such as $T_{j}$ or reduction in volume of copper. This would allow investigation into whether or not such a PCM can be identified. The density of the PCM could also be one important consideration in the choice of the cooling system in terms of weight. Other considerations may include the integrity of the foam under repeated thermal cycling and the superheating of the PCM in contact with the heat source.

Future work will be dedicated to the identification or development of PCM-based designs leading to acceptable $T_{j}$ and $t_{s}$ by increasing the accuracy of the model, which means taking into account the fins efficiency, the convection in the fluid phase or the expansion during the phase change, to name a few.

\section{REFERENCES}

[1] A.K. Jain, S. Mathapati, V.T. Ranganathan, and V. Narayanan, "Integrated starter generator for 42-V powernet using induction machine and direct torque control technique," in IEEE Trans. Power Electron., Vol. 21, no 3, pp. 701-710, 2006.

[2] S. Kaboli, E. Vahdati-Khajeh, and M.R. Zolghadri, "Probabilistic voltage harmonic analysis of direct torque controlled induction motor drives," in IEEE Trans. Power Electron., Vol. 21, no 4, pp. 1041-1052, 2006.

[3] P.T. Cheng, C.C. Hou, and J.S. Li, "Design of an auxiliary converter for the diode rectifier and the analysis of the circulating current," in IEEE Trans. Power Electron., Vol. 23, no 4, pp. 1658-1667, 2008.

[4] M. A. Juds, K. Lee, M. G. Solveson, W. E. Berkopee, and J. A. Becker, "A coupled thermal and electrical time-domain soft starter system model," in IEEE IAS Annual Meeting, Vol. 4, pp. 2607-2614, 2005. 
[5] D. Maillet, S. André, J. C. Batsale, A. Degiovanni, and C. Moyne, Thermal quadrupoles: solving the heat equation through integral transforms, Wiley, New-York, 2000.

[6] J. C. Batsale, D. Maillet, and A. Degiovanni, "Extension de la notion de quadripole thermique à l'aide de transformations intégrales: calcul du transfert thermique au travers d'un défaut plan bidimensionnel," in Int. J. Heat Mass Transfer, Vol. 37, no 1, pp.111-127, 1994.

[7] F. Wang, G. Chen, D. Boroyevich, S. Ragon, M. Arpilliere, and V.R. Stefanovic, "Analysis and design optimization of diode front-end rectifier passive components for voltage source inverters," in IEEE Trans. Power Electron., Vol. 23, no 5, pp. 22782289, 2008.

[8] F. Profumo, A. Tenconi, S. Facelli, and B. Passerini, "Instantaneous junction temperature evaluation of high-power diodes (thyristors) during current transient," in IEEE Trans. Power Electron., Vol. 14, no 2, pp. 292-299, 1999.

[9] T. Bruckner and S. Bernet, "Estimation and measurement of junction temperatures in a three-level voltage source converter," in IEEE Trans. Power Electron., Vol. 22, no 1, pp. 3-12, 2007.

[10] H. Stehfest, "Algorithm 368: Numerical Inversion of Laplace Transforms," in Comm. ACM, Vol. 13, no 1, pp. 47-49, 1970.

[11] Phase control thyristor data sheet. Eupec, Inc., Lebanon, New-Jersey. [Online]. Available: http://www.eupec.com.

[12] S. Krishnan and S. V. Garimella, "Thermal management of transient power spikes in electronics - phase change energy storage or copper heat sinks?" in ASME J. Electron. Packag., Vol. 126, no 3, pp. 308-316, 2004.

[13] V. V. Calmidi and R. L. Mahajan, "The effective thermal conductivity of high porosity fibrous metal foams," in ASME J. Heat Transfer, Vol. 121, no 2, pp. 466-471, 1999.

[14] V. Alexiades and A. D. Solomon, Mathematical modelling of melting and freezing processes, Hemisphere Publishing Corporation, USA, 1993.

[15] A. Abramowitz and I. A. Stegun, Handbook of Mathematical Functions with Formulas, Graphs, and Mathematical Tables, Dover, NY, 1964. 
TABLE I

PARAMETERS USED IN EQN. (13) IN THE CATHODE-SIDE COOLING CASE

\begin{tabular}{rccccc}
\hline $\mathrm{i}$ & 1 & 2 & 3 & 4 & 5 \\
\hline $\mathrm{R}_{i}$ & 0.02810 & 0.00106 & 0.00487 & 0.00237 & 0.0021 \\
$\mathrm{TC}_{i}$ & 4.13 & 0.45 & 0.126 & 0.0374 & 0.0091 \\
\hline
\end{tabular}

TABLE II

PARAMETERS USED IN EQN. (13) IN THE BOTH-SIDES COOLING CASE

\begin{tabular}{rccccc}
\hline $\mathrm{i}$ & 1 & 2 & 3 & 4 & 5 \\
\hline $\mathrm{R}_{i}$ & 0.00848 & 0.00243 & 0.00304 & 0.00272 & 0.00033 \\
$\mathrm{TC}_{i}$ & 0.676 & 0.132 & 0.062 & 0.0134 & 0.0019 \\
\hline
\end{tabular}


TABLE III

THERMOPHYSICAL PROPERTIES OF SELECTED MATERIALS [12]

\begin{tabular}{lcccc}
\hline & $\mathrm{Bi} / \mathrm{In} / \mathrm{Sn}$ & $\begin{array}{c}\text { Triacontane } \\
+ \text { Aluminum }\end{array}$ & $\begin{array}{c}\text { Cerrobend } \\
\text { eutectic }\end{array}$ & $\mathrm{Al}$ \\
\hline$k_{s}(\mathrm{~W} / \mathrm{mK})$ & 42.5 & 19.7 & 19 & 237 \\
$C_{p s}(\mathrm{~kJ} / \mathrm{kg} \mathrm{K})$ & 0.203 & 1814.4 & 0.167 & 24.2 \\
$k_{l}(\mathrm{~W} / \mathrm{mK})$ & 42.5 & 19.7 & 19 & - \\
$C_{p l}(\mathrm{~kJ} / \mathrm{kg} \mathrm{K})$ & 0.203 & 1814.4 & 0.167 & - \\
$\rho\left(\mathrm{kg} / \mathrm{m}^{3}\right)$ & 8060.7 & - & 9300 & 2700 \\
$T_{m}\left({ }^{\circ} \mathrm{C}\right)$ & 60 & 65 & 70 & - \\
$L(\mathrm{~kJ} / \mathrm{kg})$ & 29.5 & 251 & 32.6 & - \\
\hline
\end{tabular}

TABLE IV

PERFORMANCE OF DIFFERENT PCM-BASED HEAT SINKS IN TERMS OF RESOLIDIFICATION TIME $t_{s}$ AND JUNCTION TEMPERATURE $T_{j}$, AND COMPARISON WITH AN ALUMINUM HEAT SINK FOR AN INPUT PULSE $Q=3 \mathrm{KW}$ DURING $15 \mathrm{~S}$ AND TWO TOP COPPER BUS THICKNESSES $\left(\mathrm{W}_{1} \approx 1.9 \mathrm{CM} \mathrm{AND}_{2} \approx 0.9 \mathrm{CM}\right)$

\begin{tabular}{lccccc}
\hline Material & $\begin{array}{c}\mathrm{T}_{m} \\
\left({ }^{\circ} \mathrm{C}\right)\end{array}$ & $\begin{array}{c}\text { Volume } \\
\left(\mathrm{m}^{3}\right)\end{array}$ & $\begin{array}{c}\mathrm{T}_{j}\left(\mathrm{w}_{1}\right) \\
\left({ }^{\circ} \mathrm{C}\right)\end{array}$ & $\begin{array}{c}\mathrm{t}_{s} \\
(\mathrm{~min})\end{array}$ & $\begin{array}{c}\mathrm{T}_{j}\left(\mathrm{w}_{2}\right) \\
\left({ }^{\circ} \mathrm{C}\right)\end{array}$ \\
\hline $\mathrm{Bi} / \mathrm{In} / \mathrm{Sn}$ & 60 & 0.0002 & 83.66 & 675 & 96.35 \\
Triacontane & 65 & 0.0016 & 81.03 & 9.3 & 167.64 \\
Cerrobend & 70 & 0.0001 & 112 & 657 & 142.55 \\
Aluminum & N/A & 0.0007 & 103 & N/A & 184 \\
(current design) & & & & & \\
\hline
\end{tabular}




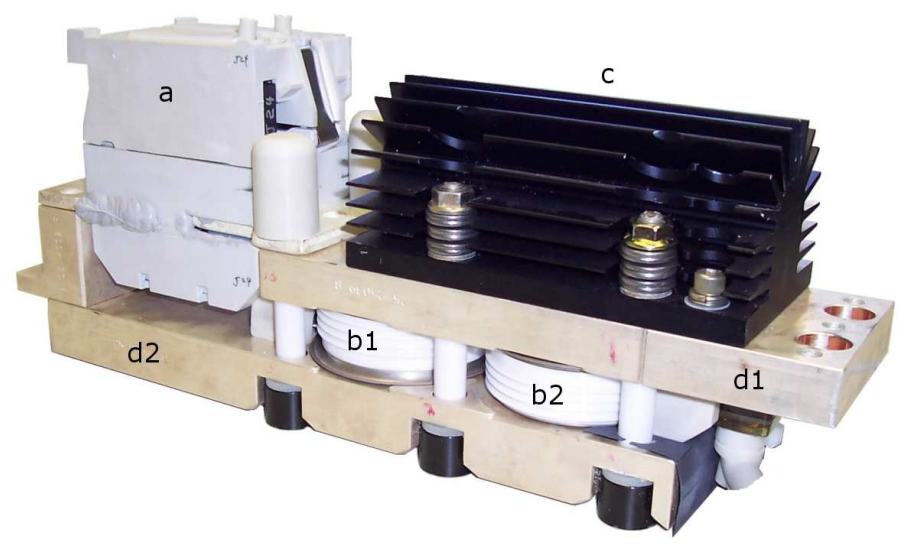

Fig. 1. Single pole of a medium voltage soft starter. Shown are the bypass contactor (a), thyristors (b1-b2), heat sink (c), and top (d1) and bottom (d2) copper buses [4].
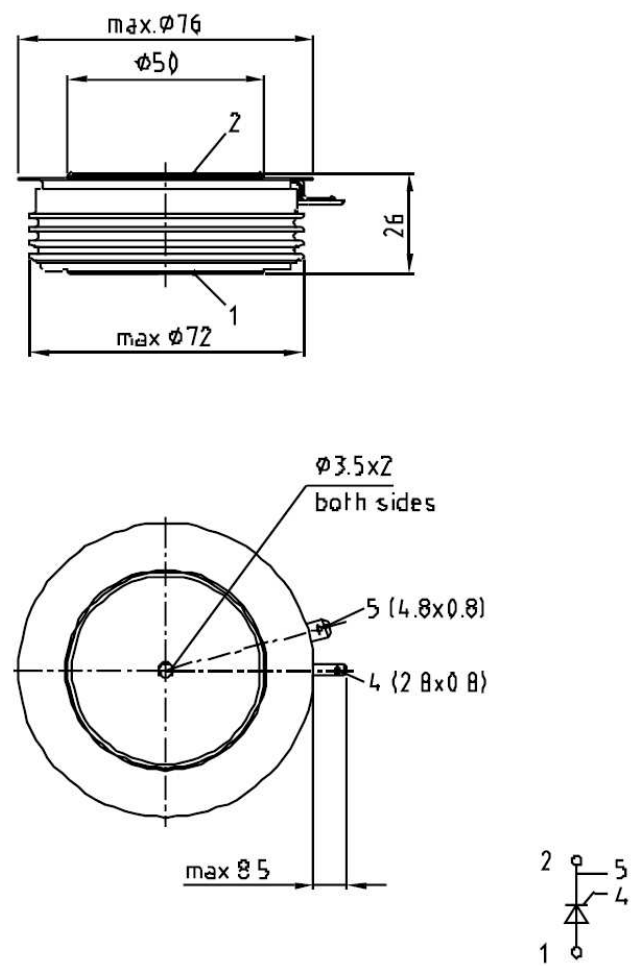

Fig. 2. Outline drawing of the T501N thyristor [4]. 


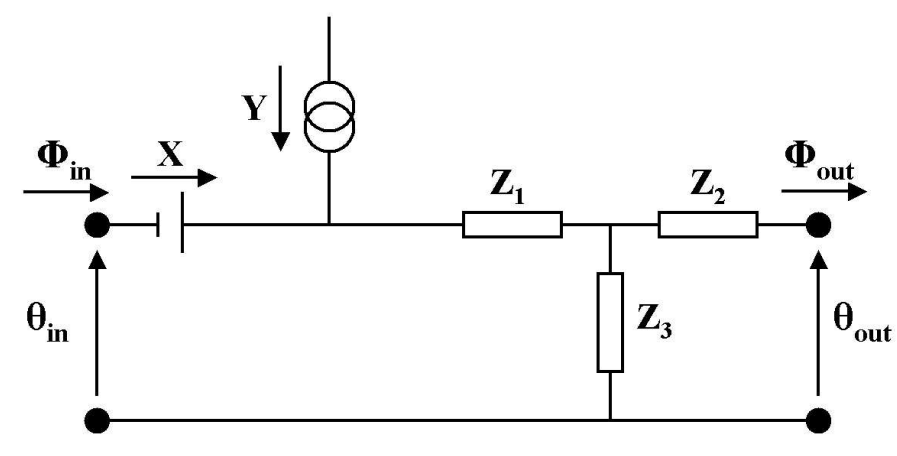

Fig. 3. Representation of the system using impedances.

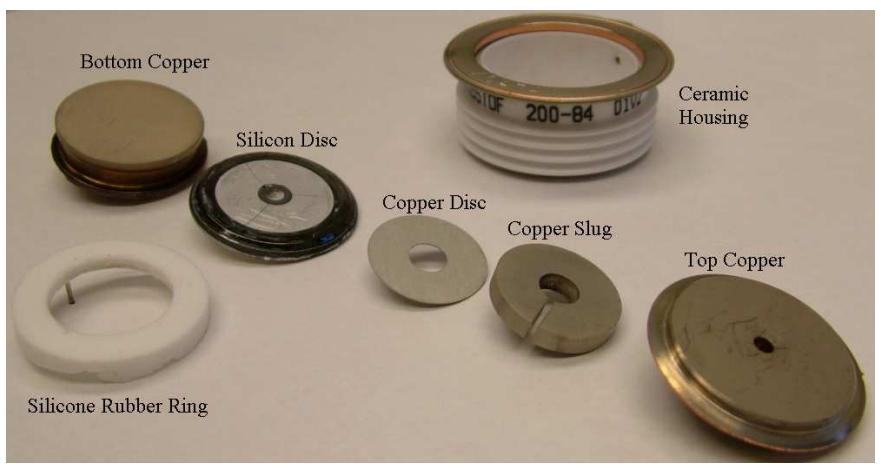

Fig. 4. Photograph of the different parts of the SCR (Eaton). 


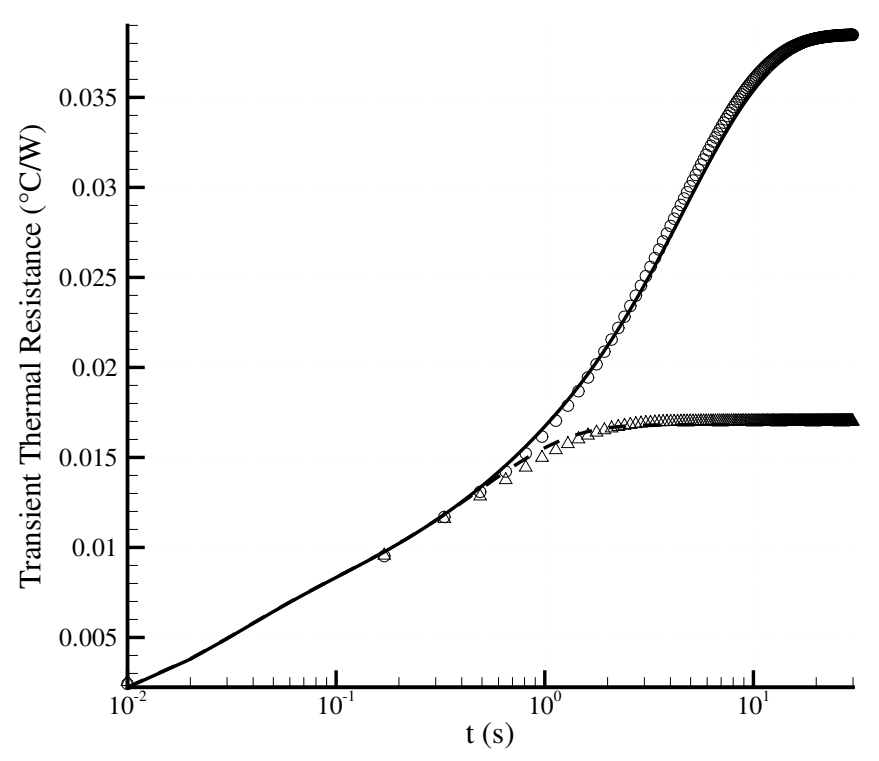

Fig. 5. Comparison between theoretical (dashed and solid lines for both-sides and cathode-side cooling, respectively) and calculated (symbols) transient thermal resistances.

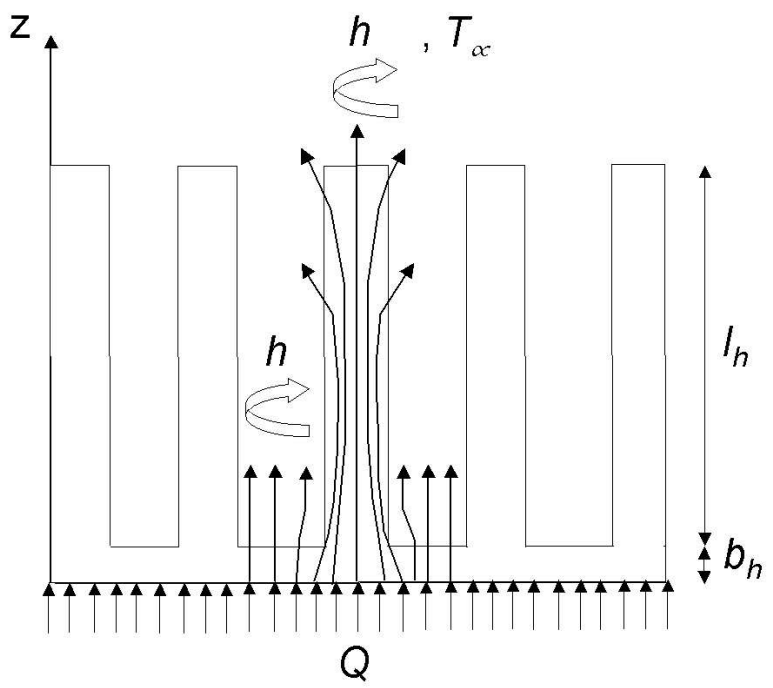

Fig. 6. Sketch of the heat flow paths in the solid-fin heat sink. 


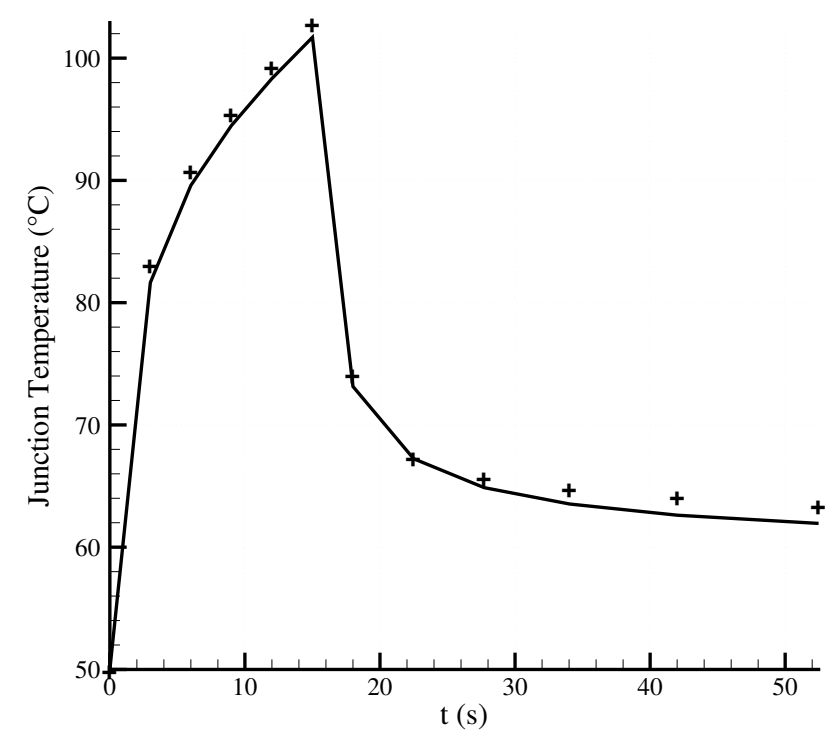

Fig. 7. Comparison between junction temperature variations calculated with an RC equivalent (solid line) and the quadrupolebased (symbols) thermal model.

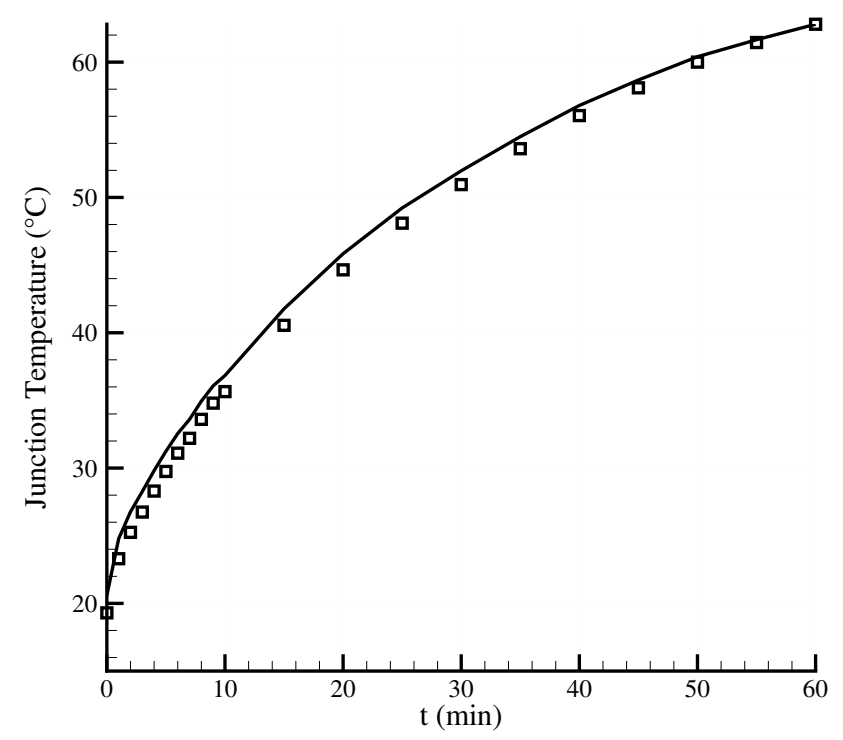

Fig. 8. Comparison between junction temperature variations measured experimentally (symbols) and calculated with the quadrupole-based thermal model (solid line). 


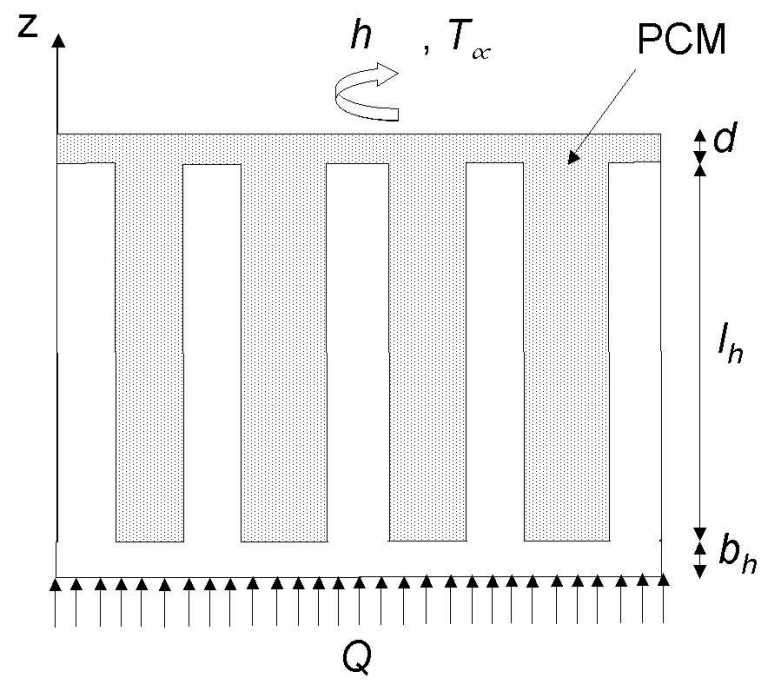

Fig. 9. Hybrid heat sink.

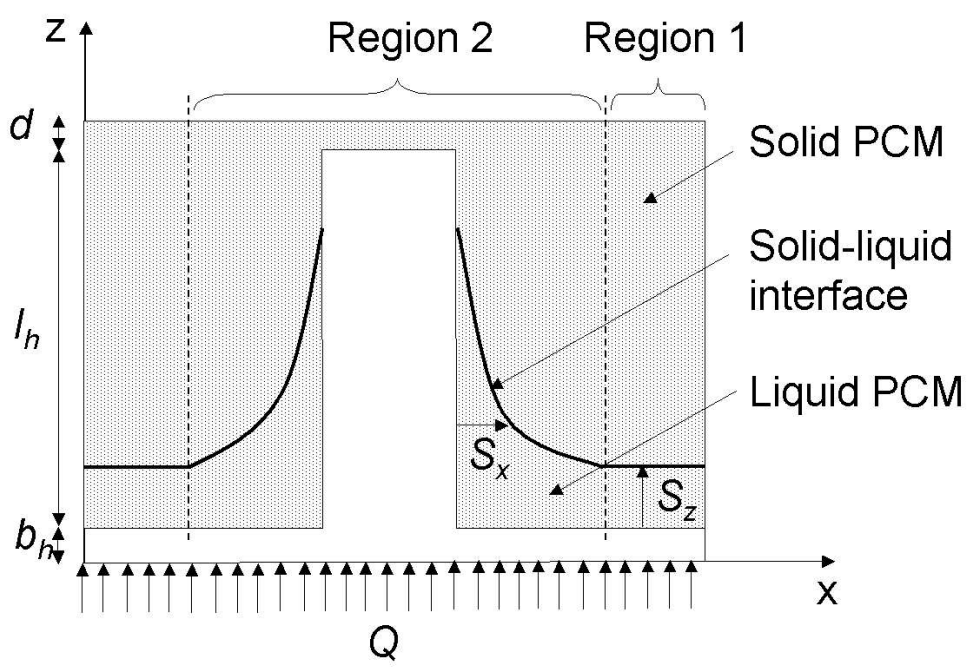

Fig. 10. Schematic of the melting front in the vicinity of the fin. 